\title{
Research on Urban Traffic Signal Control Systems Based on Cyber Physical Systems
}

\author{
Li-li Zhang $\mathbb{D}^{1,2}{ }^{1,2}$ Q Zhao, ${ }^{2}$ Li Wang, ${ }^{2}$ and Ling-yu Zhang ${ }^{2}$ \\ ${ }^{1}$ College of Information Engineering, Beijing Institute of Petrochemical Technology, Beijing 102617, China \\ ${ }^{2}$ Beijing Key Lab of Urban Intelligent Control Technology, North China University of Technology, Beijing 100144, China \\ Correspondence should be addressed to Li-li Zhang; zllphd2012@163.com
}

Received 26 June 2020; Revised 24 September 2020; Accepted 6 October 2020; Published 23 October 2020

Academic Editor: Sang-Bing Tsai

Copyright (c) $2020 \mathrm{Li}$-li Zhang et al. This is an open access article distributed under the Creative Commons Attribution License, which permits unrestricted use, distribution, and reproduction in any medium, provided the original work is properly cited.

In this paper, we present a traffic cyber physical system for urban road traffic signal control, which is referred to as UTSC-CPS. With this proposed system, managers and researchers can realize the construction and simulation of various types of traffic scenarios, the rapid development, and optimization of new control strategies and can apply effective control strategies to actual traffic management. The advantages of this new system include the following. Firstly, the fusion architecture of private cloud computing and edge computing is proposed for the first time, which effectively improves the performance of software and hardware of the urban road traffic signal control system and realizes information security perception and protection in cloud and equipment, respectively, within the fusion framework; secondly, using the concept of parallel system, the depth of real-time traffic control subsystem and real-time simulation subsystem is realized. Thirdly, the idea of virtual scene basic engine and strategy agent engine is put forward in the system design, which separates data from control strategy by designing a general control strategy API and helps researchers focus on control algorithm itself without paying attention to detection data and basic data. Finally, considering China, the system designs a general control strategy API to separate data from control strategy. Most of the popular communication protocols between signal controllers and detectors are private protocols. The standard protocol conversion middleware is skillfully designed, which decouples the field equipment from the system software and achieves the universality and reliability of the control strategy. To further demonstrate the advantages of the new system, we have carried out a one-year practical test in Weifang City, Shandong Province, China. The system has been proved in terms of stability, security, scalability, practicability and rapid practice, and verification of the new control strategy. At the same time, it proves the superiority of the simulation subsystem in the performance and simulation scale by comparing the different-scale road networks of Shunyi District in Beijing and Weifang City in Shandong Province. Further tests were conducted using real intersections, and the results were equally valid.

\section{Introduction}

Advances in technologies such as optimization algorithm [1-3], vehicle-based collaboration, hybrid driving, autonomous driving, and artificial intelligence enable researchers to develop more innovative signal control strategies and build more traffic scenarios to accommodate changes in transportation demand. At the same time, many innovative signal control strategies s not only break through the traditional signal control theory, but also propose a lot of new control logic architecture, which requires it to be tested in the field, and often involves modifying the field equipment. This project requires the close cooperation of traffic managers, control strategy researchers, information technology professionals, and implementers. It is not only time-consuming and labor-intensive, but also has certain traffic safety risks. Therefore, few traffic control systems have advanced control strategies in the past, and these control strategies have been verified in practice, such as SCOOT [4], SCATS [5], etc., and test new in such mature systems. Control strategies are also very difficult. In order to solve this problem, we propose a new urban traffic signal control and control architecture based on information physics system theory and parallel system theory and carry out thorough tests to bridge the gap 
between research and practice of traffic signal control strategies (hereinafter referred to as UTSC-CPS). China's traffic signal control field has experienced 20 years of development. Although advances have been made in the function and performance of signal control equipment, there are still major problems in the standardization of communication protocols. Different brands of traffic signal controllers and control systems are not compatible, and the traffic control language in the industry is not uniform. Not to mention the combination of VISSM [6], PARAMICS [7], and other traffic simulation software with the signal controller to achieve the control strategy [8], although there have been some attempts, the results are minimal. In sharp contrast, in North America, traffic signal controllers have adopted the NTCIP [9] protocol in a unified manner, and simulation software providers such as PTV have also embedded the most fully functional traffic signal simulator into their latest version, which not only simulates the function of the hardware traffic signal controller but also provides a comprehensive communication module conforming to the NTCIP standard [10]. Nonetheless, traditional simulation software is still very complex and difficult in parameter calibration and control strategy development. Taking into account the above issues, UTSC-CPS mainly provides a realtime simulation system, which can automatically calibrate parameters based on detection data and GIS data, can simulate macroscopic and microscopic traffic flow characteristics, and provides control strategy APIs and programming templates. The interaction between the real-time control system and the real-time simulation system facilitates the rapid test, iteration, and practice of the control strategy and provides a protocol conversion platform, which can access various types of signal controllers through the solution system and traffic control equipment, and computing and edge computing platforms to improve computing power and information security. Therefore, the UTSC-CPS system is expected to promote the research and deployment of innovative signal control strategies in future urban traffic.

The rest of this paper is organized as follows: first, the literature on the development of traditional traffic signal control system and the application of CPS in traffic management are reviewed. Then, the UTSC-CPS architecture and related technologies are elaborated. Thirdly, an intersection overflow control strategy is proposed to demonstrate the application of UTSC-CPS system. In the end, two case studies are used to verify the intersection overflow control strategy and the real-time simulation system.

\section{Literature Review}

In this section, we divide the development of urban road traffic signal control in China into four stages, namely, traffic control 1.0 (mechanization), traffic control 2.0 (electrification), traffic control 3.0 (information), and traffic control 4.0 (intellectualization), as shown in Figure 1 (the object, target, method, actuator, and detection of traffic control are listed in four stages and the development of evaluation). Among them, traffic control 1.0 is called the era of mechanization.
The first manual traffic light switch in Shanghai is the initial stage of traffic control in China [11]. Traffic control 2.0 and traffic control 3.0 are the longest and most important stages in China. The entry of SCOOT [4] and SCATS [5] systems into China in the 1980s marked the process of information of urban traffic control in China and also promoted the investment of Chinese researchers in the research and development of traffic signal control systems. After 2000, especially in recent years, the demand for urban traffic trips in China has doubled, and the mixed traffic flow characteristics with Chinese regional characteristics have emerged. Diversity of traffic demand, complexity of traffic flow, unbalance of urban development, and uncertainty of controlled objects all put forward new requirements for the theory, control strategy, and control means of urban traffic control. Especially, in today's rapid development of technologies such as big data, cloud computing, edge computing, artificial intelligence, Internet of Things, and vehicle networking, urban traffic control in China is advancing. In the era of intelligent traffic control 4.0, it emphasizes the integration of information, calculation, and control under the background of future urban traffic control. In the era of traffic control 4.0, the most obvious changes should be controlled objects, control objectives, and actuators. Among them, the controlled objects change from traffic flow to human, vehicle, and road coordination, the control objectives change from traffic safety and traffic efficiency to the feelings of traffic participants, and the actuators change from signal controller to signal controller, traffic signs, and intelligent vehicles. All these indicate that the subject of urban traffic control has changed from vehicle to human. This is the "people-oriented" concept that China has always emphasized.

2.1. CPS and Transportation System. The requirements of traffic control 4.0 for information, calculation, and control coincide with the characteristics of Information Physics System (CPS). The concept of CPS first appeared in the United States Natural Fund in 2005 [12], then quickly received the response of the Chinese and American governments and researchers, and has carried out fruitful early work in biological, military, industrial, and other fields [13-17]. The complex characteristics of urban traffic and the difficulties it faces make it the best test site and application area of CPS [18-22]. CPS plays a more and more important role in the supervision and security control function of transportation system, which will contribute to the actual control decision-making in transportation system [23]. T-CPS mainly includes the interaction between control software, communication network, and physical devices in its structure, in which network mainly refers to the integration and integration of traffic information [24-26]. T-CPS is new in expanding application. The improvement of the first-generation transportation system provides the possibility, mainly in terms of reliability, effectiveness, and transparency. Trusted computing, time predictability, and system robustness will be the focus of the design [27-31]. Considering the compatibility of traffic control 4.0 and CPS, we designed UTSC-CPS system based on CPS architecture 


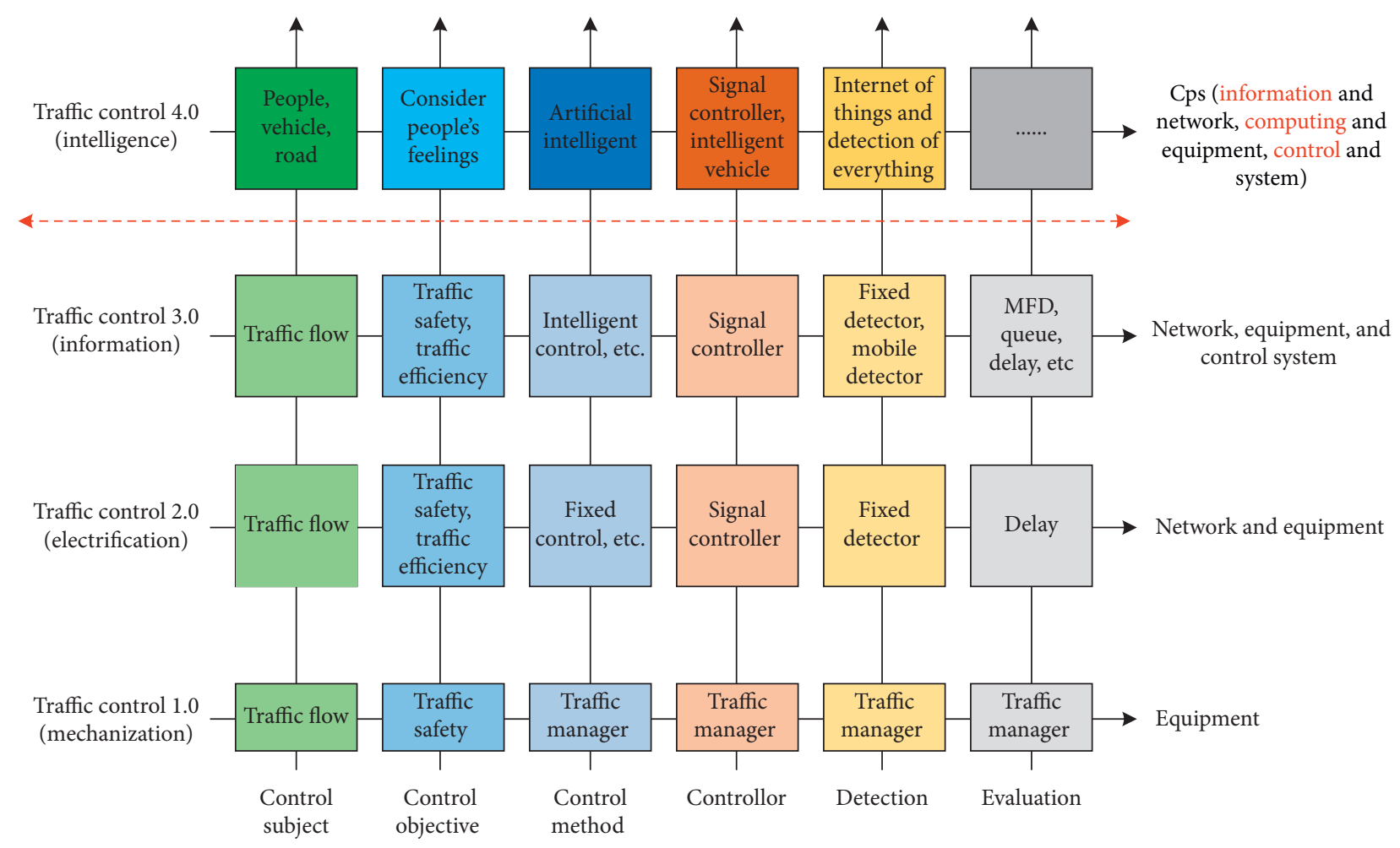

FIgURE 1: Development form of traffic control.

and fully considered the existing problems in the field of traffic signal control in China and the characteristics of future network-connected traffic. We focused on the design of protocol conversion, information security, artificial intelligence, real-time simulation, and other functions.

2.2. ACP and PTMS. While CPS is applied in the field of urban transportation, Professor Wang proposed CPSS $[32,33]$, which has a dimension expansion, based on CPS and the research results of complex systems and intelligent science, taking into account the complex characteristics of urban transportation system. Among them, Intelligent Transportation System (ITS) based on ACP [34] has put forward new concepts in traffic control modeling, experimental problems, optimization problems, and so on. It has also pushed the traditional traffic control and simulation technology to a better level and a broader perspective. This kind of research has aroused the interest of scholars all over the world. ACP [35] method can be described in the following three steps: first, considering engineering, social, human, and environmental factors as a whole, synthesizing theoretical model, empirical model, and data model, establishing an equivalent artificial system for the actual system to solve the model problem of complex system (A); through the calculation experiment of artificial system under normal and abnormal conditions, the actual system is studied. The law of interaction and evolution of elements in system (C); thirdly, connect the artificial system with the actual system, and realize parallel execution by comparing and analyzing the behavior of the two systems. System-type, reference, and forecast the future situation, and adjust the control and management methods of the two systems accordingly (P). Based on the ACP method, Professor Wang put forward the concept of parallel transportation management and control system to solve the optimization and evaluation of transportation system. By connecting the actual transportation system with the artificial transportation system, the artificial transportation experiment can be carried out, and the optimization and evaluation can be carried out conveniently. Based on ACP method, a parallel traffic management and control system PTMS $[36,37]$ is proposed. In this paper, we refer to the concept of ACP and PTMS when we design the interaction between real-time control system and real-time simulation system and visualization software and hardware in the loop system.

\section{Significance of the Research}

The innovative design of UTSC-CPS is reflected in the development, testing, simulation, and practical application of new control strategies. First, the new control strategy can be quickly implemented through the system's control strategy API, because the variables of the input and output data have been defined, and some of the control logic is also encapsulated into a function, and a programming template is provided, whether it is a researcher or a traffic manager who can quickly implement their own control strategies. Secondly, considering that traditional simulation software such as VISSIM [6] and PARAMICS [7] need a lot of manual work to complete complex parameter calibration, which is time-consuming, laborious, and error-prone, the real-time 
simulation system in UTSC-CPS provides automatic calibration function of simulation parameters based on detector data and GIS data, which can effectively solve the previous problems. Thirdly, the new control strategy can be implemented through real-time simulation. The system can be quickly optimized and upgraded. When the evaluation index meets the field application conditions, it can be quickly input into the real-time control system, without the need for professional programmers and implementation of manual implementation to the field. Fourthly, it also simulates various types of traffic scenarios, such as road congestion, green wave control, emergency management, and so on, using visual hardware and software in-the-loop system and simulation system, which enables researchers and managers to quickly analyze and design schemes. More importantly, these data are from the field detectors.

UTSC-CPS has also done a lot of work in system expansion, information security, universality, and so on. Firstly, it uses cloud computing platform to provide powerful computing and storage capabilities for control strategy and simulation. At the same time, considering that the method based on artificial intelligence will become the basis of signal control, it also provides GPU cluster computing resources for this purpose. Secondly, it does not pay attention to information security content in the traditional signal control field, when in the future vehicle network and automatic driving. When it is widely used, information security will be the key problem. Therefore, two layers of information security perception and protection are innovatively designed in the architecture of UTSC-CPS, which can effectively block network attacks targeting at signal controllers or as springboards. Thirdly, most of the communication protocols of signal controllers in China are private. Although a lot of work has been done in relevant parts of China, this phenomenon is still very common. This system has considered this problem at the beginning of the architecture and designed a protocol conversion middleware, which innovatively decouples the field equipment from the control system, so that users will not need to pay attention to the field equipment. Specific details are also provided to illustrate that the middleware has integrated NTCIP, which can be quickly applied to China and the United States and other areas of signal control.

\section{UTSC-CPS Traffic Signal Control Framework}

Considering the requirements of control, information, and computing in CPS architecture, four supporting platforms are constructed when designing and developing UTSC-CPS system. They consist of control and simulation platform, private cloud computing platform, intelligent gateway, and edge computing platform, as shown in Figure 2. (A) In the design of control and simulation platform, the idea of parallel system theory and the needs of some urban traffic managers in China are used for reference. The real-time control system and the real-time simulation system are effectively connected through the visual hardware-in-theloop system. Therefore, the real-time simulation system provides the rapid realization, verification, and optimization of new control strategies and traffic management concepts. The real-time control system provides the field execution of the verified control strategies. Visual hardware-in-the-loop system as a bridge and display way provides a macro perspective for managers and researchers to understand urban traffic. (B) Private cloud computing platform provides flexible storage, computing, and information security capabilities through virtualization technology. In particular, the UTSC-CPS system also designs GPU resources, which can provide computational support for the application of artificial intelligence technology. (C) We are particularly proud of the design of UTSC-CPS intelligent gateway, which fully takes into account the existing problems in the field of urban road traffic control devices in China. It is also based on the research results of various types of traffic signal controllers in Beijing, China, from 2008 to 2013. Through the intelligent gateway, the system and traffic control equipment are decoupled, and letters of different protocols and control concepts are made. No. 1 controller can be connected to UTSC-CPS. (D) Edge computing platform is designed as a key node to support future urban road traffic. It mainly provides information security and on-site computing capabilities. It needs to be explained that future urban intersections will be the key nodes for data aggregation of various traffic scenarios, such as automatic driving, vehicle networking, public transport, mobile travel, etc. Therefore, information security and computing capabilities on the edge side are provided. It is always important.

\subsection{Architecture and Function of UTSC-CPS}

4.1.1. Real-Time Traffic Control System. In different regions of China, there are traffic demand mainly for motor vehicles and mixed traffic demand for nonmotor vehicles and motor vehicles, which are different from the regional characteristics of traffic travel in the United States and European countries. For this reason, the real-time control system in UTSC-CPS also takes full account of this special feature and provides a variety of controlled objects and control objectives. According to the traffic demand that may arise from this feature, a variety of control strategies including regional coordinated control, trunk green wave control, singlepoint adaptive control, antioverflow control, multiperiod timing control, and special light color control are designed. In particular, it should be pointed out that the above control strategies support a variety of detection data types, including geomagnetic detectors, wide-area radar detectors, video detectors, and floating car detectors. Considering the importance of evaluation, an evaluation index set, such as imbalance index and saturation index, is designed to provide more intuitive and reasonable feedback for the evaluation of control strategies. Most importantly, the system provides verification and protection mechanism of VPN online upgrade and backup and signal control timing scheme data. The design inspiration of online upgrade comes from the design of smartphone online upgrade and the problems and experiences of some urban traffic 


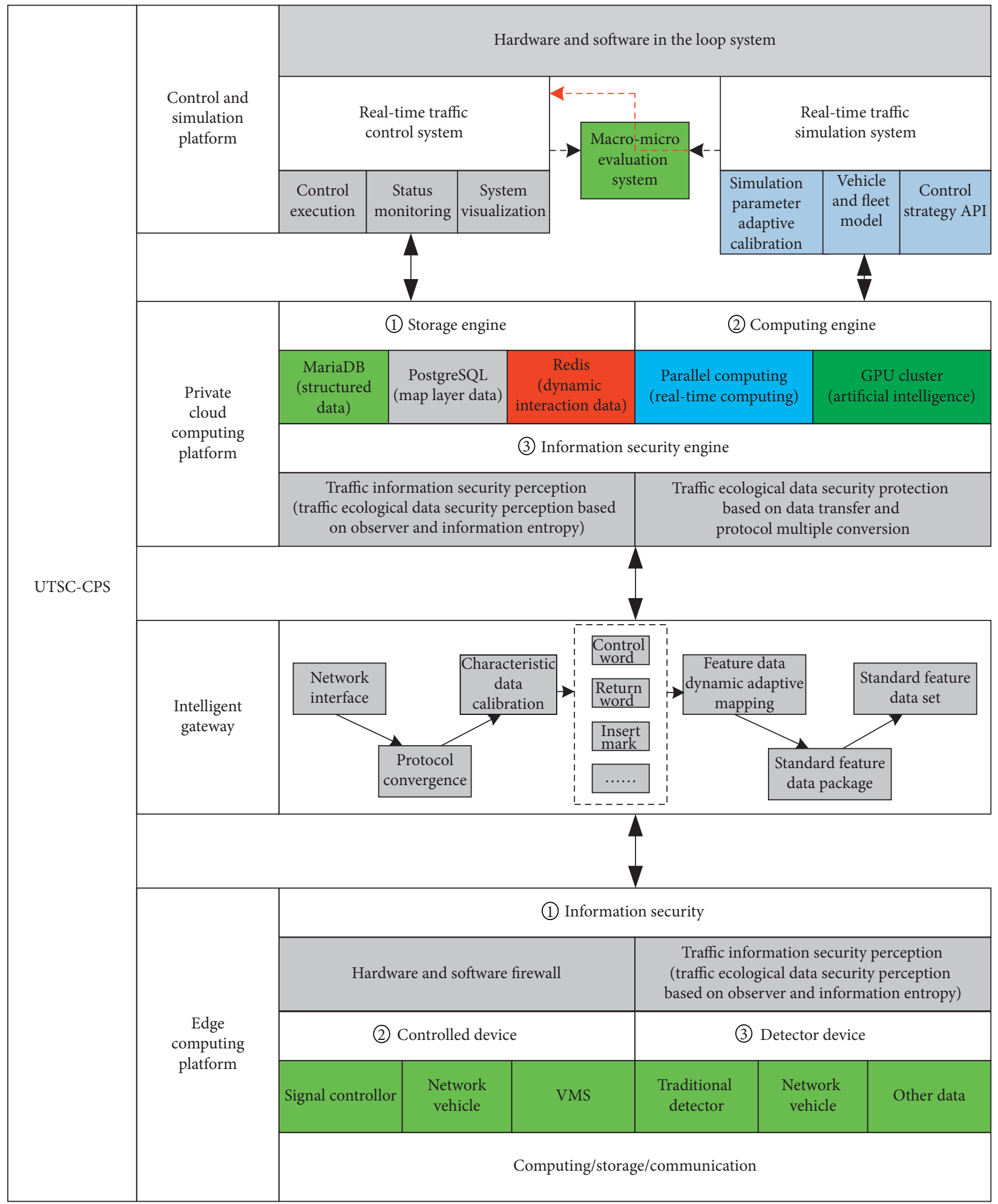

FIGURE 2: System architecture of the "UTSC-CPS" urban road traffic signal control platform.

management departments in China in the past system upgrade and the design of verification and protection mechanism of signal control timing scheme data. It can effectively prevent traffic safety problems caused by staff negligence and unprofessional timing schemes, as shown in Figures 3(a) and 3(b).
4.1.2. Real-Time Traffic Simulation System. Real-time simulation system aims at fast analysis and validation of new signal control strategies, so it is different from microsimulation software VISSIM [6], PARAMICS [7], and macroplanning simulation software TRANSCAD [38]. It mainly simulates traffic flow under the influence of control 


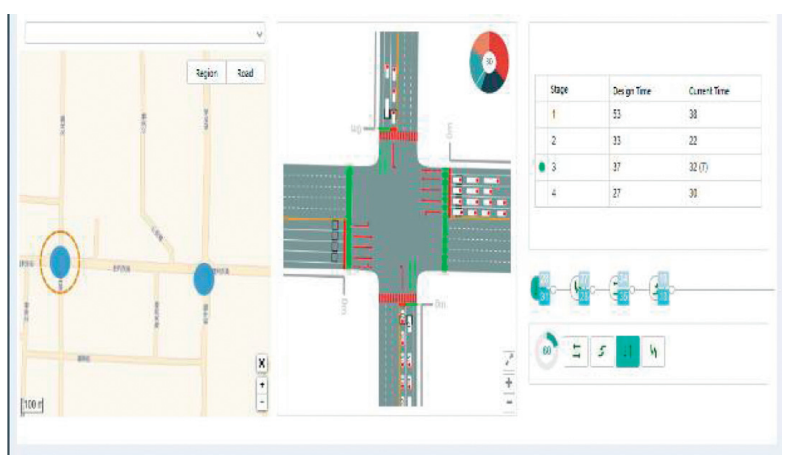

(a)

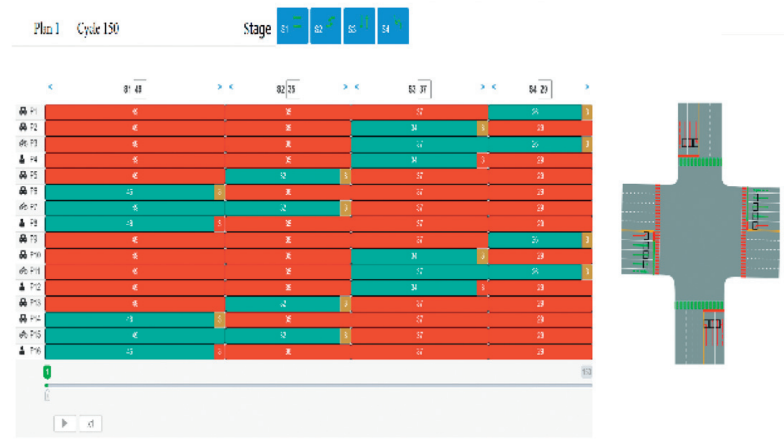

(b)

Figure 3: System architecture of the "UTSC-CPS" urban road traffic signal control platform. (a) Traffic signal control. (b) Traffic signal control plan.

strategies, so as to promote the iterative optimization of control strategies. In order to realize visualization of traffic flow, calibration of dynamic parameters of traffic flow, and calibration of basic parameters of road network in simulation, the design of simulation driver engine refers to SUMO [39] simulation software, as shown in Figures 4(a) and $4(\mathrm{~b})$.

(1) Autocalibration of Simulation Parameters. It should be emphasized that the dynamic characteristics of a single vehicle and the calibration of simulation parameters based on manual work are neglected actively in the design of the simulation system. The system uses detector data and GIS data to automatically calibrate static data (road properties: road length, lane width, intersection shape, etc.) and dynamic data (vehicle type ratio, velocity distribution, steering ratio, headway time, and so on). At the same time, traffic flow characteristics and car-following characteristics are based on the accumulation of historical detection data, and real-time detection data are corrected.

(2) Control Strategy Architecture and API Design. Real-time simulation system provides good support for the development of new control strategies through the architecture shown in Figure 5. This architecture consists of three parts: data preparation, control strategy, and equipment engine. (A) The data preparation part encapsulates the test data as standard input and output variables and stores the signal control, vehicle, and variable sign as control variables in the database. (B) In the control strategy part, traffic demand is first constructed into different virtual scenes. Control strategies are designed for different scenarios. The set of control strategies is called agent, and control strategies are called agent. That is to say, the new control strategy designed by researchers is defined as agent, and the corresponding agent is run through given conditions in the simulation system. (C) The device engine part realizes the interaction between the real-time simulation system and the real-time control system. When the new control strategy is proved to be reliable, it can be pushed directly into the real-time control system through the engine and executed in the real environment at the right time. We call the implementation of this control strategy architecture Scene Driven, Software Definitions (SD), have encapsulated a wealth of functions in the software, and designed a standard programming template, so that managers and researchers without programming technology can quickly implement their own control strategies and be verified, as shown in Figure 6.

\subsubsection{Visual Hardware and Software in the Loop System.} In ancient and modern wars of China, military experts were good at carrying out war deduction through sand table and carrying out corresponding strategies and tactics. Referring to this form and idea of combat, we design a visual hardware-software in-the-loop system for urban road traffic control as shown in Figure 7, in which the real-time control system and the real-time simulation system are its foundation. The system can simulate traffic control and road state. The data used in the system can be real-time data on the spot, making it a mapping of real traffic. At the same time, it can also use real-time simulation system data to make it a presentation of simulated traffic. Urban traffic managers can discover and screen traffic control effects from a macro perspective and can also construct different traffic scenarios to test new control strategies, as shown in Figure 8(a). It should also be noted that the cellular automata model is also designed in the architecture of the system, which can provide researchers with simulation based on cellular automata, as shown in Figure 8(b).

\subsubsection{Cloud Computing and Edge Computing Platform}

Computing Power Output. The complexity of urban traffic is reflected in the variability of traffic demand, the dynamic nonlinearity of traffic flow, and the randomness of traffic participants. Considering the large-scale and scalable computing power required by control strategies and other core algorithms, we design a cloud computing platform based on virtualization, which can provide the required storages

At the same time, considering that edge devices and controlled individuals will be integrated into the system in 


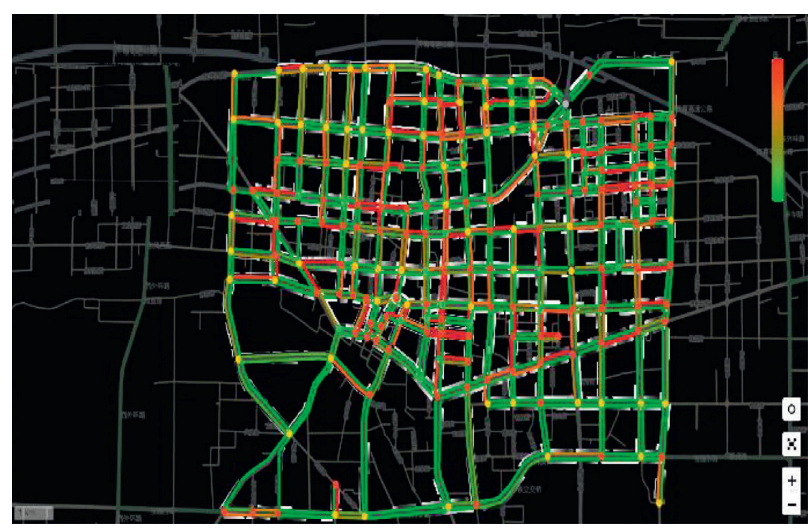

(a)

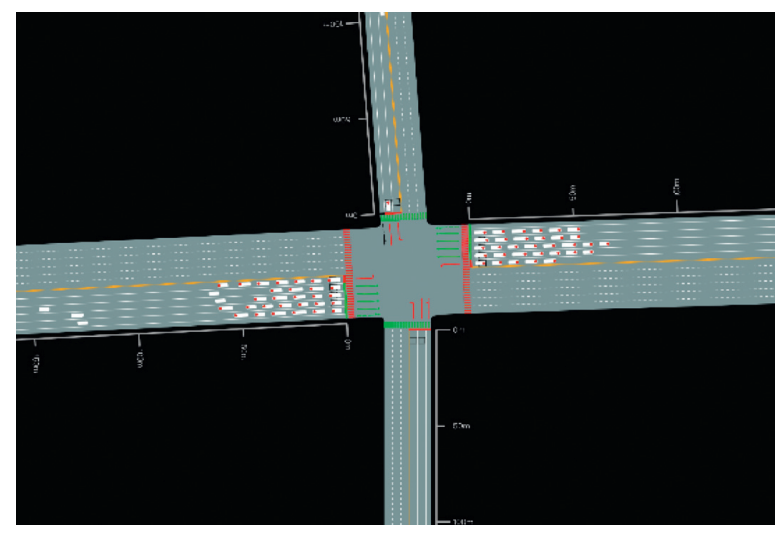

(b)

FIgURE 4: Real-time simulation functional structure design. (a) Simulation of road network. (b) Simulation of intersection.

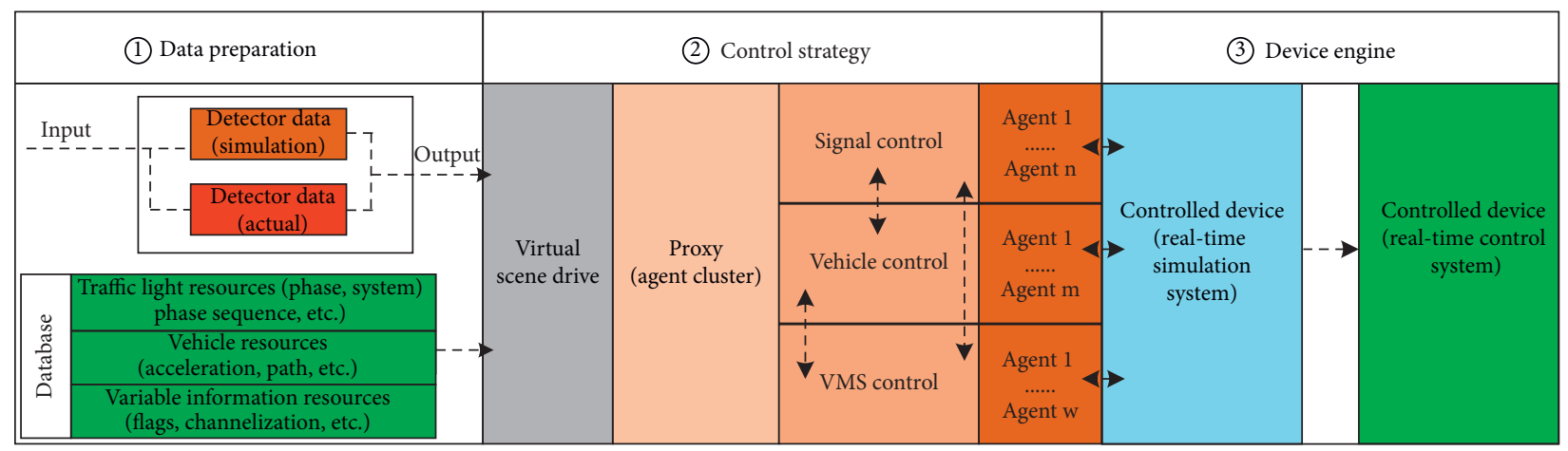

FIGURE 5: Control strategy API architecture of real-time simulation system.

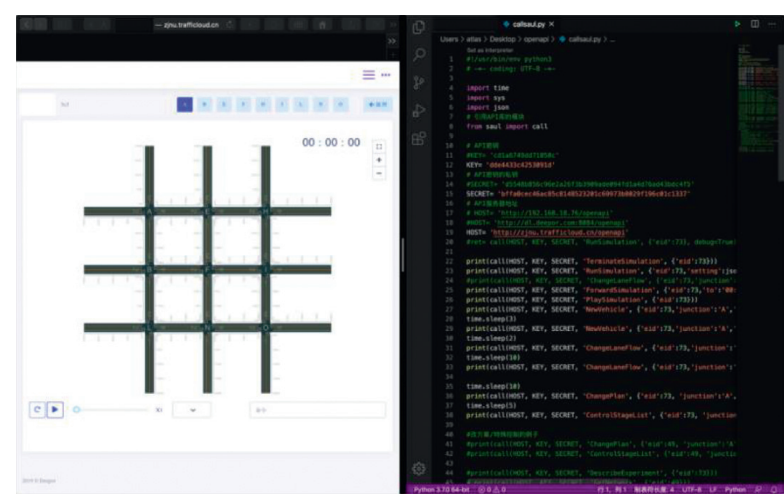

FIGURE 6: Control strategy software diagram.

the future, and the key technology of centralized large data processing based on cloud computing model cannot satisfy the efficient processing of data generated by edge devices, four key issues are focused on the following: (A) linear growth of centralized cloud computing capability cannot match explosive growth of massive edge data; (B) slave network as network edge devices transmit massive data to the cloud center, resulting in a sharp increase in the load of network transmission bandwidth, resulting in long network delay; (C) network edge data involve personal privacy, making privacy security issues become particularly prominent; and (D) network edge devices with limited power transfer data to the cloud center consume larger power. In order to solve the above four key problems, edge computing platform and cloud computing platform are designed.

It is noteworthy that AI technology has been applied in urban traffic field, such as traffic flow forecasting, image processing, automatic driving, etc. AI technology for urban traffic control and management is also being studied. Therefore, when we design UTSC-CPS architecture, we also fully consider the support of AI and can provide GPU cluster computing power output and edge meter for it. Computing power can be output for system applications.

What is urban traffic control? In 2010, Iran's nuclear facilities were attacked by Stuxnet [34], which proved to be the first virus specifically targeting the real world infrastructure. Since then, this kind of attack has been well known from the background to the front and has also brought new challenges to information security. Vehicles are controlled by hackers in "The Fate of the Furious" [31]. The scene of hitting targets from all directions has also become a highlight of the film, but it also arouses our concern. Intelligent Traffic Signal Control (ITC), as an important node equipment for 


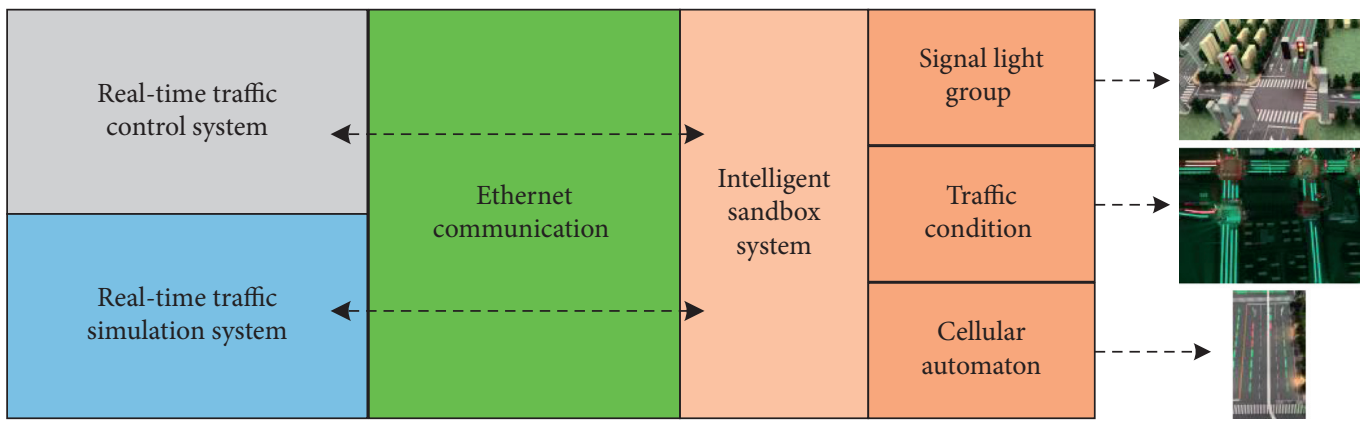

Figure 7: Visualized hardware and software in the loop system architecture.

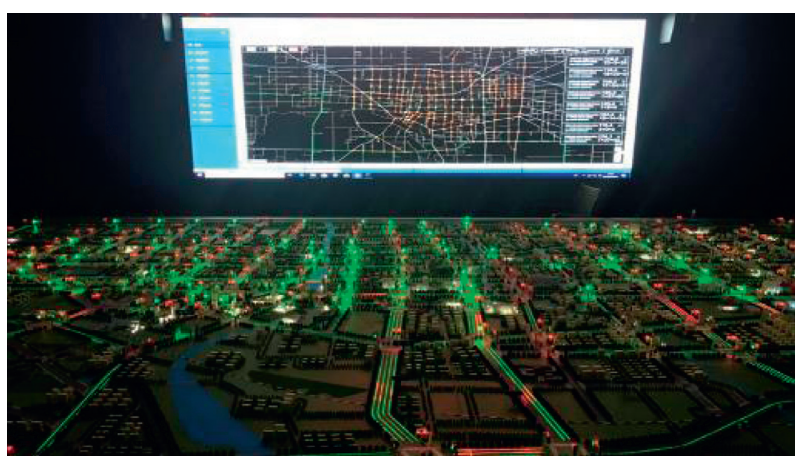

(a)

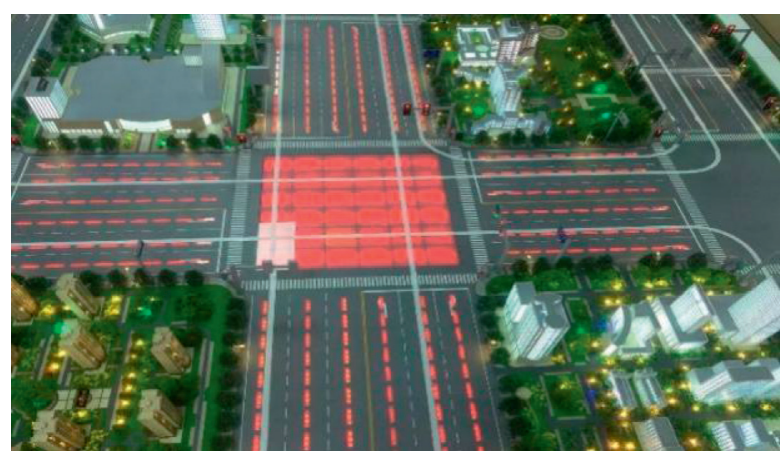

(b)

Figure 8: Visualized hardware and software in the loop system of Weifang City in Shandong Province, China. (a) Visualized hardware and software in the loop system. (b) Cellular automata.

urban road traffic linking traffic participants, not only undertakes the implementation of control strategies, but also acquires and transmits all kinds of traffic in real time. T-Type Traffic Information. Under the background of Internet traffic, the safety of input and output data, which is the key node, must be paid attention to. Taking the signal controller as an example, it can be divided into four categories (as shown in Figure 9): (A) the signal controller as the main body is attacked directly (not through other extended devices); (B) the extended device is the springboard, and the attack signal controller (detector); (C) the external device is the springboard, and the attack signal controller (vehicle networking-signal controller); and (D) the signal controller is the springboard, and the attack signal controller is the springboard. Hit the peripheral equipment (signal controller-vehicle networking). Therefore, in the framework of UTSC-CPS, the importance of information security is considered in both cloud computing platform and edge computing platform, and the ways of information security perception and protection are designed and developed. Among them, the combination of data state perception and information entropy is used in information security perception, and the firewall of hardware and software is used in protection mode. However, with the change and deepening of hacker attack mode and technology, the future urban traffic information security will become a key issue for researchers and managers, because it is not only related to government security, but also related to government security. It is about the safety of traffic participants.

4.1.5. Intelligent Gateway of UTSC-CPS. UTSC-CPS intelligent gateway not only provides powerful network throughput capability for traffic signal controller, but also provides access protocol aggregation and standard conversion functions for various detection and control devices. In order to ensure that the network connection needs to meet the transmission time certainty and data integrity, the unified technical standard time-sensitive networking (TSN) [40, 41] defined by IEEE for key services such as real-time priority and clock is adopted. At present, the interface and protocol of detection and control equipment in China's traffic field are not unified, which brings great difficulties for equipment access and data use. For this reason, the control plane of the network is decoupled from the data forwarding plane by using software-defined networking $(\mathrm{SDN})[40,41]$ design to realize the programmable control of convergence and conversion of multi-nonstandard protocols, as shown in Figures 10 and 11.

\subsection{Technical Characteristics of UTSC-CPS}

(1) Coexistence and Covariance of Virtual and Real. UTSC-CPS should have the functions of real-time monitoring and real-time online simulation of 


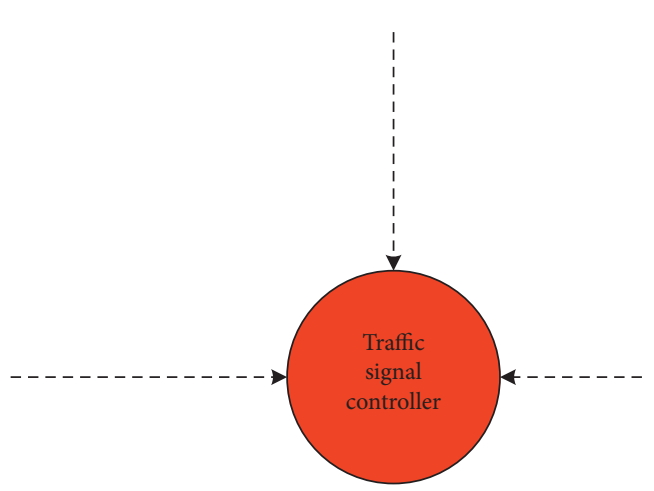

(a)

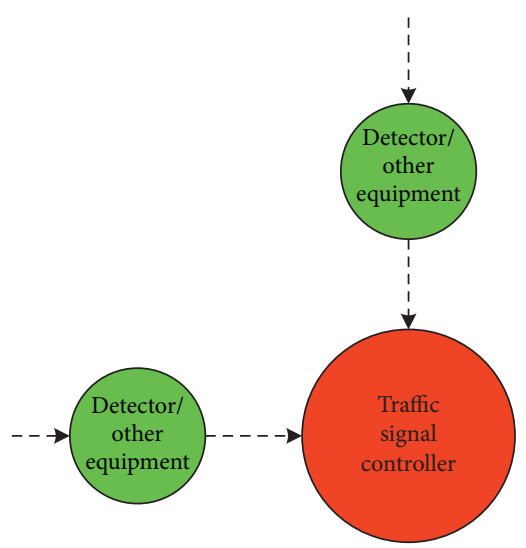

(c)

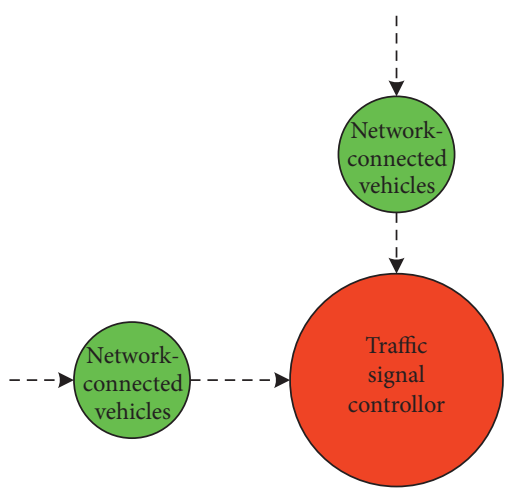

(b)

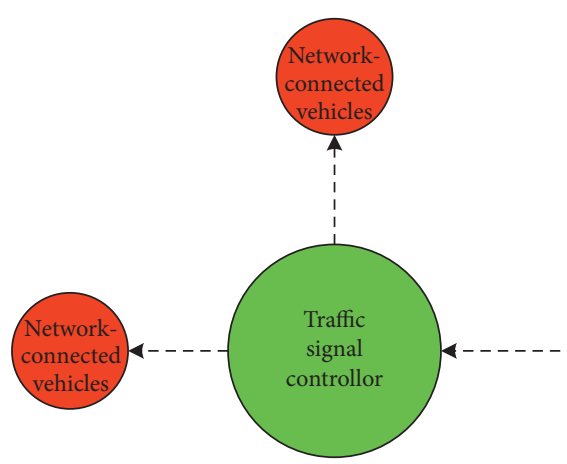

(d)

Springboard for attack

Target of attack

- - Flow of attack data

Figure 9: Classification of information security attack scenarios of traffic field equipment.

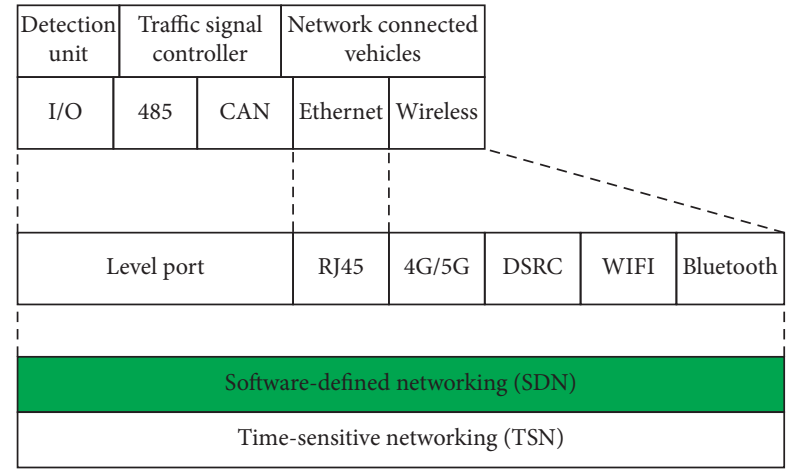

FIgURE 10: Structure of intelligent gateway.

information system and physical system based on urban road traffic control. Data-driven UTSCCPS uses real-time information from physical systems to automatically calibrate and modify simulation models and parameters to improve simulation accuracy; simulation results affect the behavior of physical systems through UTSC-CPS control.
(2) Multi-to-Multi Dynamic Connection. UTSC-CPS system links traffic elements including people, vehicles, roads, and environment through large-scale heterogeneous networks to form a multilayer information network to realize the interaction and sharing of dynamic information.

(3) Real-Time Parallel Computing and Information Processing. UTSC-CPS system framework includes multisource data fusion and processing, large-scale online real-time data-driven simulation, large-scale distributed computing, and other requirements, so it is necessary to use cloud computing to build a macroelastic computing platform and use edge computing to build a microdedicated computing platform to meet the requirements of UTSC-CPS centralized control and decentralized control.

(4) Self-Organization, Self-Adaptation, Self-Diagnosis, and Self-Healing. Facing the future, a large number of mobile terminals, including people and vehicles, will quickly access UTSC-CPS system, which requires UTSC-CPS system to have self-organizing and self-adaptive functions. At the same time, the increasingly large and complex system should have 


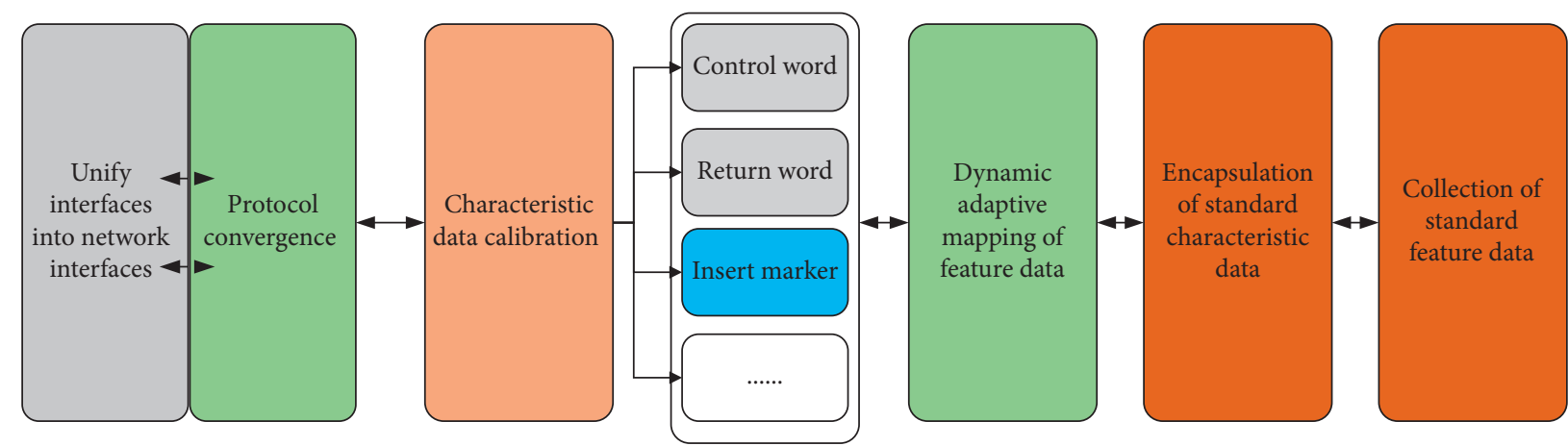

FIGURE 11: SDN-protocol interface aggregation and conversion.

self-diagnosis and self-healing capabilities in the face of various failures and information security.

\subsection{Key Technologies of UTSC-CPS}

(1) Collaborative Technology of Global Optimization and Local Control. The combination of centralized control and decentralized control to achieve the optimal coordination of global optimization and local control is the first technical problem to be solved by UTSCCPS.

(2) Large-Scale Distributed Computing and Layered Information Security. Cloud computing and edge computing collaboration: cloud computing effectively integrates the computing, storage, and communication resources of the system, realizes the distributed computing requirements of large-scale macrosystems, and uses edge computing to realize fast computing, communication, and control of data measurement. At the same time, information security perception and protection are realized at cloud end and edge side, respectively, effectively blocking attacks based on communication.

(3) UTSC-CPS Communication Protocol. In order to realize the deep integration of information system and physical system, a new UTSC-CPS communication protocol is needed to meet the requirements of computing synchronization, data management, and information transmission.

(4) Dynamic Networks and Delay/Interrupt-Tolerant Networks. In order to meet the requirements of UTSC-CPS for reliability and speed of online computing and analysis, it is necessary to enhance the ability of communication network to handle delay, packet loss, and interruption and construct dynamic network and delay/interrupt-tolerant network.

(5) Automatic Mapping Consistency in Virtual and Real Spaces. It is an important function of UTSC-CPS to realize the comprehensive analysis and simulation of information system and physical system. The consistency of automatic mapping of virtual and real space includes (1) ensuring the synchronization and consistency of real-time information of the system with the actual situation and (2) ensuring the accuracy of simulation model and results.

(6) Cooperation between UTSC-CPS and vehicle cyber physical system (V-CPS): the goal of UTSC-CPS control system with traffic control as its core should be combined with that of V-CPS control system, and the integrated management of urban road traffic in the future can be realized through information sharing and collaborative control.

\section{Experiment and Analysis}

5.1. Simulation Study on Weifang City in Shandong Province and Shunyi District in Beijing. In order to simulate the scale, real-time performance, stability, and reliability of the realtime simulation system, different-scale urban road network and traffic flow were used to test the system.

(i) Select two different levels of urban road network and traffic flow for simulation test. In order to ensure authenticity and accuracy, the simulation network and fleet data are provided by relevant departments in Shunyi District of Beijing and Weifang City of Shandong Province, as shown in Figures 12(a) and 12(b).

(ii) In order to test the real-time performance and stability of the platform, the refresh rate of the simulation is $1-125 \mathrm{~ms}$ (refresh rate is set according to the transmission frequency of the geomagnetic detector data provided by the relevant departments to ensure the authenticity of the simulation), running continuously for 24 hours.

(iii) In Table 1, the hardware and software parameters of cloud computing platform based on VMware virtualization and the scale of simulation road network and traffic flow in Shunyi District of Beijing and Weifang City of Shandong Province are given in Table 2.

Simulated road network diagram: according to the traffic flow data provided by the relevant departments in Shunyi District of Beijing and Weifang City of Shandong Province, the simulation load of the fleet is correlated with the traffic time (morning peak, evening peak, and night), and the 


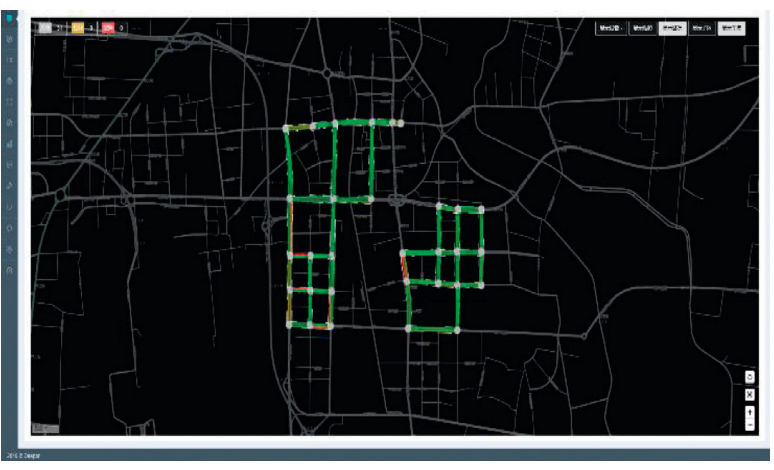

(a)

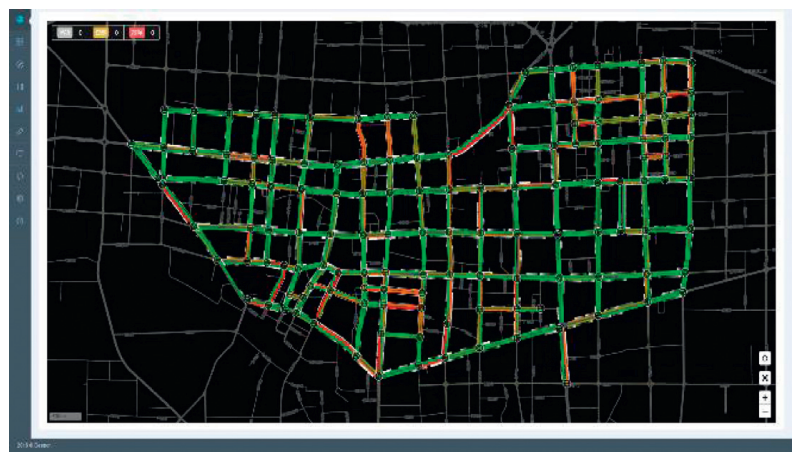

(b)

FIGURE 12: Simulation test road network. (a) Shunyi District road network of Beijing. (b) Weifang City road network of Shandong.

TABLE 1: VMware virtualization of cloud hardware and software parameters.

\begin{tabular}{lc}
\hline Name & Configuration requirements \\
\hline CPU & Two Intel 8-core Xeon E7-4809 V4 processors $(2.1 \mathrm{GHz}, 20 \mathrm{M}$ cache) \\
RAM & Two memory $64 \mathrm{~GB}(4 \times 16 \mathrm{~GB}) 2133 \mathrm{MHz} \mathrm{DDR} 4$ \\
Storage & Standardized $82.5^{\prime \prime}$ SAS hot swap hard disk slots, $8 * 300 \mathrm{~GB}$ \\
Network card & Four Realtek RTL8111F $10 / 100 / 1000 \mathrm{Mbs}$ \\
Virtualization & VMware ESXI6.5 + Vphere6.5 \\
Operating system & CentOS6.8_64_min_base \\
Development & Lua5.1+C + python3 + PHP7.0 \\
\hline
\end{tabular}

TABLE 2: Simulation of road networks and traffic flow scales.

\begin{tabular}{lcccc}
\hline Position & Intersection scale & Road scale & Traffic flow scale & Refresh rate (ms) \\
\hline Shunyi District & 31 & 84 & 252 & 125 \\
Weifang City & 134 & 446 & 1624 & 125 \\
\hline
\end{tabular}

Explanation: the fleet size is modeled as a medium fleet. The number of vehicles represented by the fleet is converted to the maximum detection length of the geomagnetic detector, which is about 10-30 vehicle size.

refresh rate of the simulation platform and the CPU load capacity of the server are tested, respectively. The test results are displayed in Table 3.

As shown in Figures 13(a) and 13(b) above, the refresh ratio (actual refresh time/125 ms) of simulation platforms with different sizes of intersections, sections, and fleets can be found. With the increase of fleet size, the refresh ratio of simulation platforms increases, i.e., the actual refresh time increases, which indicates that the performance of simulation platforms decreases, but it can still be accepted. Figures 13(c) and 13(d) show the CPU resources required by the simulation platform. The private cloud platform can support large-scale simulation requirements by mobilizing computing power and release computing power quickly when the simulation demand decreases to achieve dynamic load balancing adjustment.

5.2. Case Study: Real Intersection Test. In order to test and prove the reliability and advanced nature of the system, a one-year construction and application was carried out with the support of the Weifang City Traffic Detachment in Shandong Province, and the following example tests were conducted based on the single intersection. Modeling and simulation of static data such as lanes, road sections, and canalization at intersections are completed. Calibration of simulation dynamic data uses detection data of wide-area radar, as shown in Figure 14(a). Among them, the selected detection data is workday data and covers morning peak (7: 30-8:30), flat peak (10:00-11:00), and evening peak (17: 30-18:30); traffic scenarios include four types: undersaturated, oversaturated, undersaturated and transit, and oversaturated and transit. In the programming template provided by the UTSC-CPS system, the detector data and traffic control data (phase, phase sequence, phase, interval time, green light time, lane, period, etc.) have been defined as variables, and the timing control has also been defined., Multiperiod control, induction control, space-time resource allocation control, and traffic scene recognition methods are packaged into functions for direct call, as shown in Figure 14(b). For the test intersection of Shengli East Road and Fushou Street, four control strategies of static-phase sequence multiperiod control, static-phase sequence induction control, dynamic-phase sequence control, and dynamic resource scheduling control were mainly formulated and analyzed. 
TABLE 3: Simulation platform performance test results.

\begin{tabular}{|c|c|c|c|c|c|c|c|}
\hline \multicolumn{8}{|c|}{ Shunyi District of Beijing } \\
\hline Time & 7:00-9:00 & 9:01-11:30 & $11: 31-13: 00$ & $13: 01-16: 30$ & $16: 31-19: 30$ & $19: 31-22: 30$ & $22: 31-6: 59$ \\
\hline Traffic flow scale & 240 & 152 & 177 & 120 & 250 & 210 & 80 \\
\hline Refresh rate $(\mathrm{ms})$ & 10.3 & 8.1 & 8.5 & 7.6 & 12.5 & 10 & 3.2 \\
\hline CPU load capacity (\%) & 25 & 17 & 18 & 10 & 27 & 22 & 6 \\
\hline \multicolumn{8}{|c|}{ Weifang City of Shandong Province } \\
\hline Time & 7:00-9:00 & 9:01-11:30 & $11: 31-13: 00$ & $13: 01-16: 30$ & $16: 31-19: 30$ & $19: 31-22: 30$ & $22: 31-6: 59$ \\
\hline Traffic flow scale & 1580 & 1092 & 1290 & 1100 & 1620 & 1310 & 820 \\
\hline Refresh rate $(\mathrm{ms})$ & 82.3 & 62.2 & 70.1 & 65 & 88.5 & 72.4 & 54.2 \\
\hline CPU load capacity (\%) & 55 & 44 & 48 & 45 & 57 & 50 & 41 \\
\hline
\end{tabular}

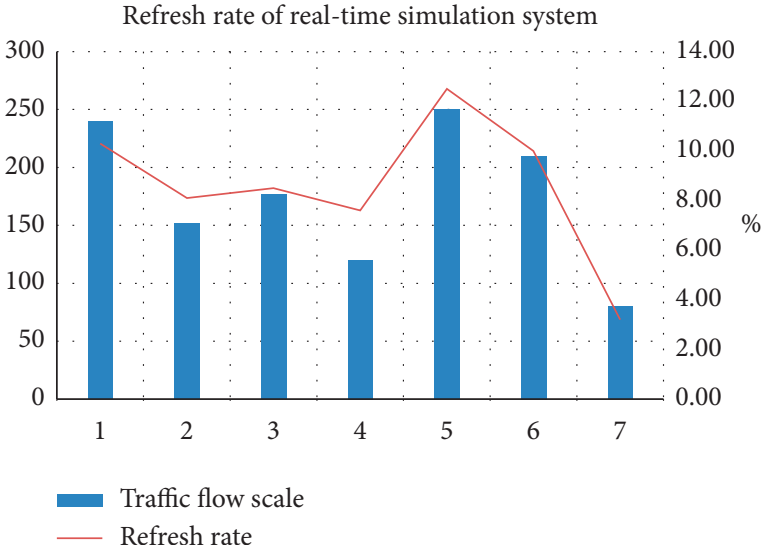

(a)

CPU load capacity of real-time simulation

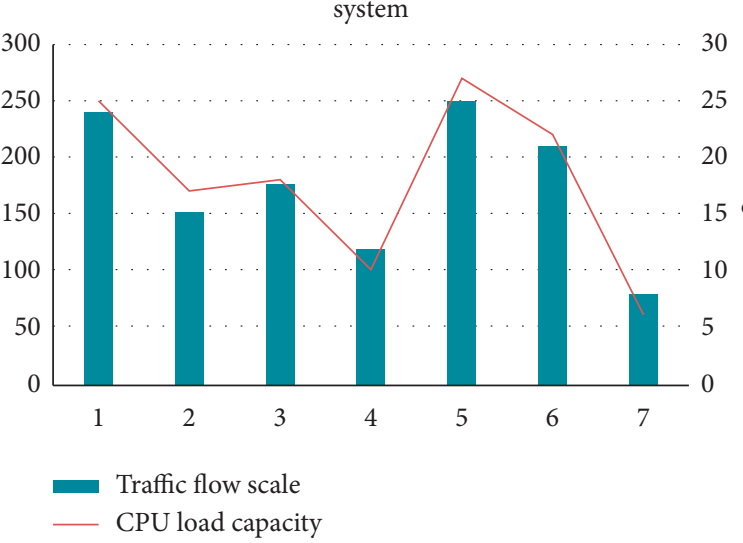

(c)

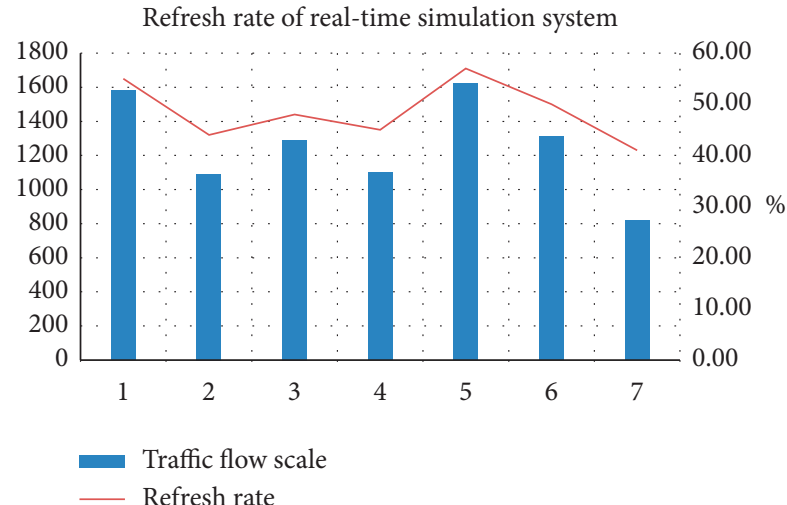

(b)

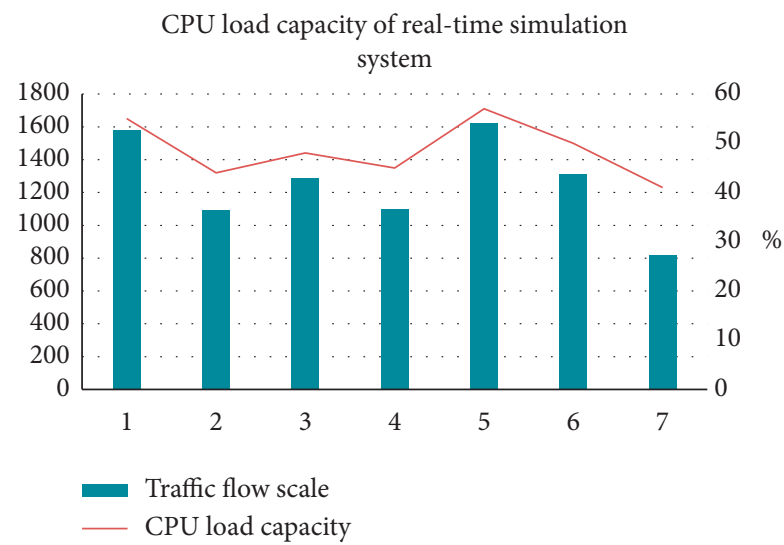

(d)

FIGURE 13: Simulation platform performance test results.

Simulate the four-phase control of static phase sequence multiperiod control (SSMTC), static-phase sequence induction control (SSATC), dynamic-phase sequence control (DSTC), and space-time resource allocation control (DRSTC) at the test intersection and use UTSC-CPS. The three evaluation indexes (through rate, average parking, and queuing length) designed in the system (as shown in Figure 15) analyze and evaluate the simulation control effects under the four control modes. The simulation time for each period is 3600 s, and the number of simulations is 10 times. Since the simulation used in this article is directly generated from the raw data of the wide-area radar, there is no need to consider the initial time in traditional simulation software. After the simulation, data sorting and analysis are performed. The evaluation index data is the arithmetic average of ten simulation results. Figure 15 shows the comparison of the three types of evaluation indexes of the four control methods of the test intersection in the morning peak, flat peak, and evening peak hours.

Note: the direct pass rate indicates the proportion of buses passing through intersections without stopping; the average number of stops indicates the average number of stops for all vehicles at the intersection within 5 minutes; the 


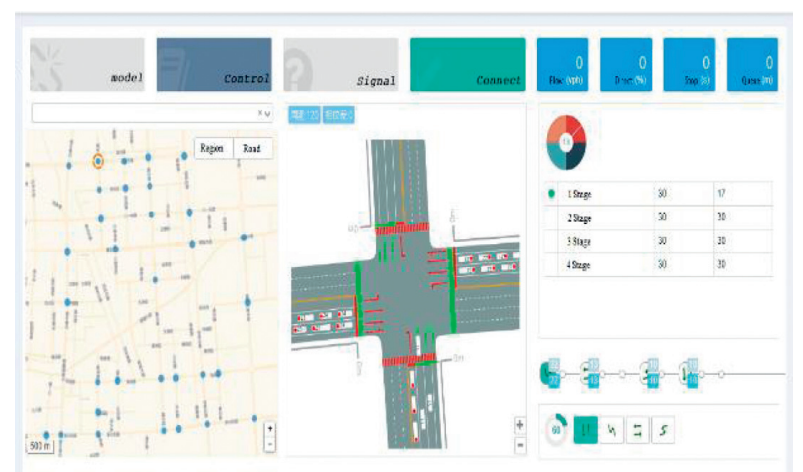

(a)

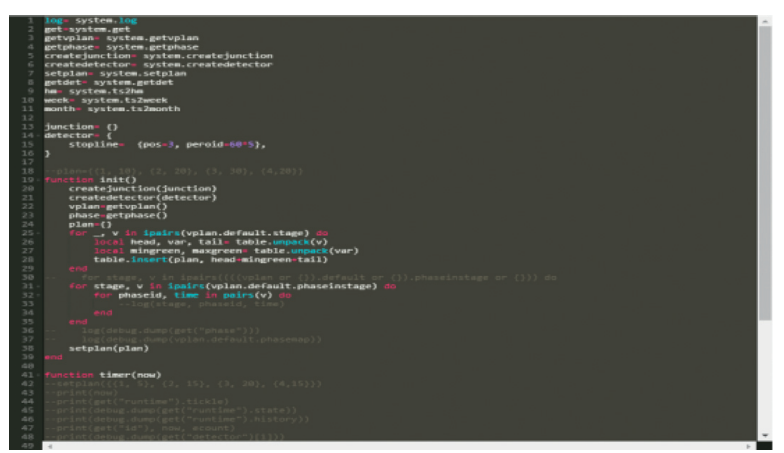

(b)

Figure 14: Intersection test. (a) Model of road network. (b) Algorithm implementation and simulation.

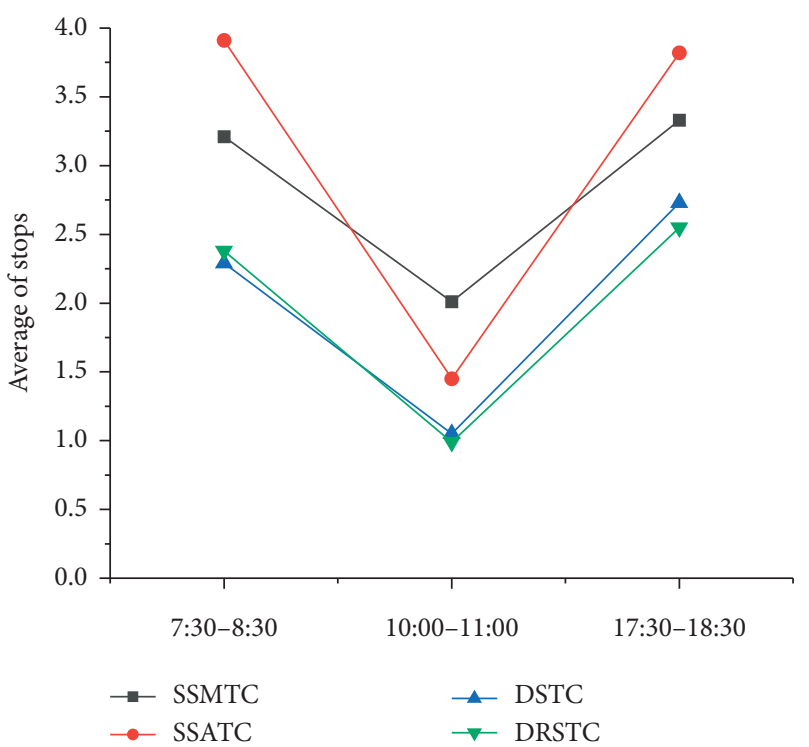

(a)

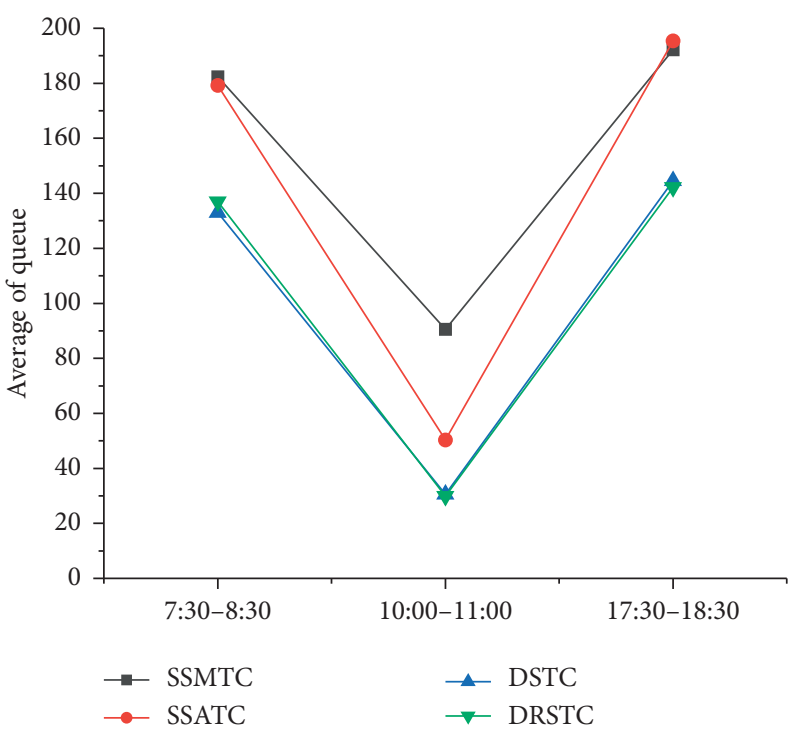

(b)

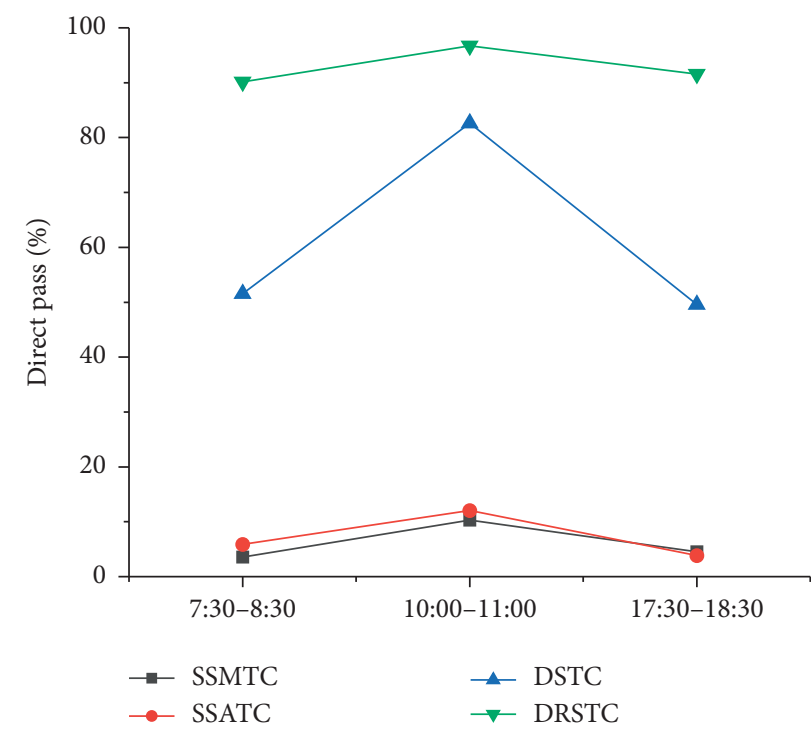

(c)

FIGURE 15: Comparison of evaluation indicators of four control methods. (a) Comparison of average of stops. (b) Comparison of average of queue. (c) Comparison of direct pass rate. 
queue length indicates the average queue length of all lanes within 5 minutes at the intersection.

From Figures 15(a) and 15(b), it can be seen that the average number of stops and the average queue length at the intersection when using the two control strategies of DSTC and DRSTC are significantly lower than those of the two control strategies of SSMTC and SSATC. However, it is worth noting that the SSATC strategy only outperforms the SSMTC strategy during average peak times. This is because the static-phase sequence multiperiod control scheme adopted in this chapter is derived from the actual operation plan of the test intersection, and the plan is optimized. It can be seen from Figure 15(a) that when the DRSTC control strategy is adopted, the bus pass rate is greatly improved compared to the other three control strategies. The reason is that DRSTC can accurately identify "undersaturated and bus" and through the scene recognition method "oversaturation and bus" two scenarios, and when these two scenarios appear, priority control can be provided through lane control. Although the DSTC control strategy can also accurately identify the scene, due to the limitation of the lane control cannot be implemented, the direct rate can only be improved by dynamically adjusting the phase sequence; the two control strategies SSMTC and SSATC cannot recognize the above two scenarios, and intersections that do not adopt public transport priority control, the pass rate is not comparable. Buses may also pass through intersections without stopping.

\section{Conclusion Remark and Future Work}

In this paper, the urban road traffic signal control information physical system UTSC-CPS is proposed. The system expands the shortcomings of current traffic signal control in control strategy development, testing, simulation, and practical applications and has made innovative work in system extension capabilities, information security, and universality.

UTSC-CPS uses the parallel system concept to establish the interaction mechanism between the real-time control system and the real-time simulation system. It bridges the research and practice of traffic signal control and fully considers the complex characteristics of urban road traffic, through the design of visual hardware and software. In the ring system to simulate a variety of traffic scenarios, the level of traffic management is improved. It also fully considers the requirements of control, information, computing, and simulation under the information physics system architecture and designs cloud computing and edge computing platforms to provide computing power, storage capacity, and information security, especially in the future network communication background. The next is more important.

Compared with other traffic signal control systems, this paper has achieved breakthroughs in three problems. First, through the self-developed real-time simulation system, the data-based parameter adaptive calibration is realized, and the real-time simulation system and real-time control system are constructed. The general underlying data architecture. Second, through the definition of good input and output data variables, packaged control logic functions are provided to researchers through programming templates, enabling them to quickly implement control strategies and use real-time online simulation systems for verification and upgrade Optimization. Third, the protocol middleware is a universal system that can adapt to most signal controllers in China and the United States without changing its physical structure.

In order to test and prove the advantages of UTSC-CPS, it was built and applied for one year with the help of the traffic detachment of Weifang City, Shandong Province, China. The results from the current results are good. At the same time, in order to verify the availability of the simulation system developed by ourselves, the performance and stress tests of the simulation system were carried out using the road network of Weifang City and Beijing Shunyi District, and the results also proved that they can be used. Further tests were conducted using real intersections, and the results were equally valid.

Although we mainly introduce the architecture and features of UTSC-CPS in this article, there are still many problems in the specific application. For this, more traffic scenarios and longer tests are needed, and more practice is needed to explore their potential and explore innovative traffic control measures.

\section{Data Availability}

The actual verification and test of the system are supported by Weifang Traffic Police Detachment in Shandong Province and the Beijing Shunyi District Traffic Police Detachment. The data provided were for use in this paper only because it involves commercial and government requirements for the use of data. As a result, the data cannot be made public. However, online access to the system can be provided. Technical details of the system can be made available upon request to the authors. The following is the access address of the system and team e-mail: http://dl.deepor.com:8086/ auth/login and http://dl.deepor.com:8088/auth/login and e-mail: zllphd2012@163.com.

\section{Conflicts of Interest}

The authors declare that they have no conflicts of interest regarding the publication of this paper.

\section{Authors' Contributions}

Conceptualization was carried out by L.L.Z. and Q.Z.; methodology was conducted by L.L.Z. and Q.Z.; resources were provided by L.W.; writing of the original draft was carried out by L.L.Z.; reviewing and editing were done by Q.Z. and L.Y.Z.; visualization was performed by L.Y.Z.; and supervision was done by L.W.

\section{Acknowledgments}

This research was supported by grants from the Beijing Municipal Great Wall Scholar Program (CIT \& TCD 20190304), the National Key R\&D Program of China under 
Grant nos. 2017YFC0821102 and 2017YFC0822504, and the Beijing Municipal Natural Science Foundation under Grant no. 4194078 .

\section{Supplementary Materials}

The actual verification and test of the system are supported by the Weifang Traffic Police Department in Shandong Province and the Beijing Shunyi District Traffic Police Department. The data provided were for use in this paper only because it involves commercial and government requirements for the use of data. (Supplementary Materials)

\section{References}

[1] Z. Zhou, S. Lin, W. Du, and H. Liang, "Integration of regional demand management and signals control for urban traffic networks," IEEE Access, vol. 7, pp. 20235-20248, 2019.

[2] D. Liu, W. Yu, S. Baldi et al., "A switching-based adaptive dynamic programming method to optimal traffic signaling," IEEE Transactions on Systems, Man, and Cybernetics: Systems, 2019.

[3] Z. Zhou, B. De Schutter, S. Lin et al., "Two-level hierarchical model-based predictive control for large-scale urban traffic networks," IEEE Transactions on Control Systems Technology, vol. 25, no. 2, pp. 496-508, 2016.

[4] P. B. Hunt, D. I. Robertson, R. D. Bretherton et al., "SCOOTA traffic method of coordinating signals," Laboratory Report No. LR, The National Academies of Sciences, Engineering, and Medicine, Washington, DC, USA, 1981.

[5] A. G. Sims and K. W. Dobinson, "The Sydney coordinated adaptive traffic (SCAT) system philosophy and benefits," IEEE Transactions on Vehicular Technology, vol. 29, no. 2, pp. 130-137, 1980.

[6] PTV VISION: VISSIM 5, 40-User Manual, PTV Group, Karlsruhe, Germany, 2013.

[7] Quadstone, Paramics 4.2 User Manual, Quadstone Ltd, Edinburgh, Scotland, 2003.

[8] S. Baldi, I. Michailidis, V. Ntampasi, E. Kosmatopoulos, I. Papamichail, and M. Papageorgiou, "A simulation-based traffic signal control for congested urban traffic networks," Transportation Science, vol. 53, no. 1, pp. 6-20, 2019.

[9] "The national transportation communications for ITS protocol," 2013, http://www.ntcip.org/.

[10] inAsc/3 Software in the Loop Controller-asc/3 silEconolite Group, Inc., Anaheim, CA, USA, 2013, https://www.econolite. com/files/1214/1384/0766/2014-Product-Suite-Controllers. pdf.

[11] "The traffic lights have a history of hundreds of years. Who invented the first signal light in the world? https://baijiahao. baidu.com/s?id=1616556025696965492\&wfr=spider\&for $=p c$.

[12] CPS Steering Group, "Cyber-physical systems executive summary," June 2011, http://precise.seas.upenn.edu/events/ iccps11/doc/CPS-Executive-Summary.pdf.

[13] J. R. Wang, M. Q. Wu, and J. F. Su, "Cyber-physical system," Acta Automatica Sinica, vol. 38, no. 4, pp. 507-517, 2012.

[14] P. Derler, E. A. Lee, and A. S. Vincentelli, "Modeling cyberphysical systems," Proceedings of the IEEE, vol. 100, no. 1, pp. 13-28, 2012.

[15] Y. Tan, M. C. Vuran, and S. Goddard, "Spatio-temporal event model for cyber-physical systems," in Proceedings of the 29th IEEE International Conference on IEEE Distributed
Computing Systems Workshops, 2009. ICDCS Workshops'09, pp. 44-50, Montreal, Canada, June 2009.

[16] R. A. Thacker, K. R. Jones, C. J. Myers et al., "Automatic abstraction for verification of cyber-physical systems," in Proceedings of the 1st ACM/IEEE International Conference on Cyber-Physical Systems, ACM, Stockholm Sweden, pp. 12-21, April 2010.

[17] M. C. Bujorianu, M. L. Bujorianu, and H. Barringer, "A unifying specification logic for cyber-physical systems," in Proceedings of the 17th Mediterranean Conference on Control and Automation, 2009. MED'09, IEEE, Thessaloniki, Greece, pp. 1166-1171, June 2009.

[18] S. Jianjun, W. Xu, G. Jizhen, and C. Yangzhou, "The analysis of traffic control cyber-physical systems," Procedia - Social and Behavioral Sciences, vol. 96, pp. 2487-2496, 2013.

[19] H. Liu, W. D. Sun, and W. Liu, "Lattice hydrodynamic model based traffic control: a transportation cyber-physical system approach," Physica A: Statistical Mechanics and Its Applications, vol. 461, pp. 795-801, 2016.

[20] Y. Gong and S.-j. Li, "Fusion framework of urban traffic control and route guidance based on cyber-physical system theory," Journal of Highway and Transportation Research and Development (English Edition), vol. 7, no. 1, pp. 82-89, 2013.

[21] Y. Gong and S. J. Li, "Fusion framework of urban traffic control and route guidance based on CPS theory," Journal of Highway and Transportation Research and Development, vol. 29, no. 5, pp. 114-120, 2012.

[22] Z. H. Wang, Y. J. Zhang, and K. Du, "Traffic CPS architecture design," Journal of Highway and Transportation Research and Development, vol. 8, no. s1, pp. 142-14, 2012.

[23] E. M. Clarke, B. Krogh, A. Platzer et al., "Analysis and verification challenges for cyber-physical transportation systems," in Proceedings of the National Workshop for Research on High-Confidence Transportation Cyber-Physical Systems: Automotive, Aviation and Rail, National Science Foundation, Washington, DC, USA, pp. 20-25, November 2008.

[24] A. Tiwari, "Formal verification of transportation cyber physical systems," in Proceedings of the National Workshop for Research on High-Confidence Transportation Cyber-Physical Systems: Automotive, Aviation and Rail, National Science Foundation, Washington DC, USA, pp. 30-35, November 2008.

[25] N. Gaddam, G. S. A. Kumar, and A. K. Somani, "Securing physical processes against cyber attacks in cyber-physical systems," in Proceedings of the National Workshop for Research on High-Confidence Transportation Cyber-Physical Systems: Automotive, Aviation and Rail, National Science Foundation, Washington, DC, USA, pp. 26-29, November 2008.

[26] C. Pu, "Intelligent, integrated, and intermodal transportation services," in Proceedings of the National Workshop for Research on High-Confidence Transportation Cyber-Physical Systems: Automotive, Aviation and Rail, National Science Foundation, Washington, DC, USA, pp. 51-54, November 2008.

[27] C. Wakter, B. L. Valley, P. Ellis et al., "Dependable automotive cyber-physical system," in Proceedings of the National Workshop for Research on High-Confidence Transportation Cyber-Physical Systems: Automotive, Aviation and Rail, National Science Foundation, Washington, DC, USA, pp. 60-64, November 2008.

[28] F. Zhang and W. Wolf, "Cyber-physical control for future transportation systems," in Proceedings of the National Workshop for Research on High-Confidence Transportation 
Cyber-Physical Systems: Automotive, Aviation and Rail, National Science Foundation, Washington, DC, USA, pp. 115119, November 2008.

[29] R. Cartwright, A. Cheng, P. Hudak et al., "Cyber-physical challenges in transportation system design," in Proceedings of the National Workshop for Research on High-Confidence Transportation Cyber-Physical Systems: Automotive, Aviation and Rail, National Science Foundation, Washington, DC, USA, pp. 220-224, November 2008.

[30] S. Ghiasi, "Design methodologies for synthesis and execution of cyber-physical systems," in Proceedings of the National Workshop for Research on High-Confidence Transportation Cyber-Physical Systems: Automotive, Aviation and Rail, National Science Foundation, Washington, DC, USA, pp. 210215, November 2008.

[31] M. Iqbal and H. B. Lim, "A cyber-physical middleware framework for continuous monitoring of water distribution systems," in Proceedings of the 7th ACM Conference on Embedded Networked Sensor Systems, ACM, Berkeley, CA, USA, pp. 401-402, November 2009.

[32] F.-Y. Wang, "The emergence of intelligent enterprises: from CPS to CPSS," IEEE Intelligent Systems, vol. 25, no. 4, pp. 85-88, 2010.

[33] W. Guo, Y. Zhang, and L. Li, "The integration of CPS, CPSS, and ITS: a focus on data," Tsinghua Science and Technology, vol. 20, no. 4, pp. 327-335, 2015.

[34] F.-Y. Wang, "Toward a paradigm shift in social computing: the ACP approach," IEEE Intelligent Systems, vol. 22, no. 5, pp. 65-67, 2007.

[35] F. Zhu, G. Li, Z. Li, C. Chen, and D. Wen, "A case study of evaluating traffic signal control systems using computational experiments," IEEE Transactions on Intelligent Transportation Systems, vol. 12, no. 4, pp. 1220-1226, 2011.

[36] F.-Y. Wang, "Parallel control and management for intelligent transportation systems: concepts, architectures, and applications," IEEE Transactions on Intelligent Transportation Systems, vol. 11, no. 3, pp. 630-638, 2010.

[37] F. Zhu, Z. Li, S. Chen, and G. Xiong, "Parallel transportation management and control system and its applications in building smart cities," IEEE Transactions on Intelligent Transportation Systems, vol. 17, no. 6, pp. 1576-1585, 2016.

[38] Caliper Corporation, TransCAD User's Guide, Caliper Corporation, Newton, MA, USA, 2000.

[39] D. Krajzewicz, G. Hertkorn, C. Rössel et al., "SUMO (Simulation of Urban MObility)-an open-source traffic simulation," in Proceedings of the 4th middle East Symposium on Simulation and Modelling (MESM20002), pp. 183-187, Sharjah, UAE, 2002.

[40] Edge Computing Consortium, "White paper of edge computing consortium," 2016, http://www.ecconsortium.org/ Lists/index/cid/11.html.

[41] Edge Computing Consortium, "Edge computing reference framework 2.0," 2017, http://www.ecconsortium.org/Lists/ index/cid/11.html. 